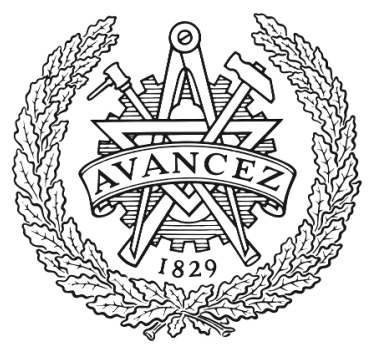

CHALMERS

UNIVERSITY OF TECHNOLOGY

\title{
A Parameterized Turnout Model for Simulation of Dynamic Vehicle-Turnout Interaction with an Application to Crossing Geometry
}

Downloaded from: https://research.chalmers.se, 2023-04-26 14:29 UTC

Citation for the original published paper (version of record):

Pålsson, B. (2020). A Parameterized Turnout Model for Simulation of Dynamic Vehicle-Turnout Interaction with an

Application to Crossing Geometry Assessment. Lecture Notes in Mechanical Engineering: 351-358. http://dx.doi.org/10.1007/978-3-030-38077-9_41

N.B. When citing this work, cite the original published paper. 


\title{
A parameterized turnout model for simulation of dynamic vehicle-turnout interaction with an application to crossing geometry assessment
}

\author{
Björn A. Pålsson ${ }^{1[0000-0002-2237-8560]}$ \\ ${ }^{1}$ Chalmers University of Technology/CHARMEC, Hörsalsv. 7A, 412 96, Göteborg, Sweden \\ bjorn.palssonechalmers. se
}

\begin{abstract}
This paper presents a parameterized structural track model for the simulation of dynamic vehicle-turnout interaction in a multi body simulation environment. The model is demonstrated by performing simulations for different vehicle speeds, crossing geometries and fixations between crossing rail and sleepers with different stiffness. Results are presented for dynamic wheel-rail contact forces, bending moments in crossing rail and sleepers and sleeper-ballast contact pressure. The main conclusions are that a) the peak dynamic bending moment in the sleeper under the crossing transition is significantly higher with a stiff direct fixing compared to a softer indirect fixing and $b$ ) the structural loading in terms of bending moment in the crossing rail, bending moment in the underlaying sleeper and sleeper-ballast contact pressure increases proportionally and significantly with increased impact angle and vehicle speed for wheels passing over the crossing transition.
\end{abstract}

Keywords: Track model, turnout, parameterization, multi body dynamics, structural loading

\section{Introduction}

Turnouts (switches and crossings, S\&C) are a critical part of railway networks as they allow for trains to change between tracks. They are also critical in the sense that rail discontinuities in the switch and crossing panels induce higher dynamic loads and greater degradation rates compared to plain line. There is therefore a demand for simulation models with predictive capability that can be used to generate input to maintenance strategies and system optimization.

One effort to improve the capability of simulation models for turnouts is the development of a so-called Whole System Model (WSM) simulation scheme within the EUproject Shift2Rail [1]. The objective of this scheme is that it should be able to assess the influence of individual components and parameters on overall turnout performance. This paper presents a partial result from these efforts; a parameterized structural turnout model for the simulation of dynamic vehicle-turnout interaction. The model is 
demonstrated by performing simulations for different vehicle speeds, crossing geometries and fixations between crossing rail and sleepers.

\section{Modeling requirements and solution}

Starting from the purpose of the Whole System Modeling scheme, two main requirements were identified for the model in addition to predictive capability. It needs to be flexible in the sense that it should allow for the simulation of a wide variety of turnout designs. It also needs to provide the output of relevant structural responses to allow for the computation of damage criteria for components in the turnout structure.

The developed solution is a Matlab script that can generate structural (finite element) turnout models for ballasted track from a set of input parameters. The scripting allows for flexibility in the model generation while the structural representation allows for the calculation of relevant damage criteria for assessment of overall turnout performance. Examples of damage criteria that can be calculated are rail and sleeper bending moments and sleeper-ballast contact pressures.

\section{$3 \quad$ Generation of turnout models}

The script generates finite element turnout models where rails and sleepers are modelled using beam elements while rail fastenings and ballast stiffness are modelled using discrete Kelvin bushing elements (linear springs and dash pots). The output from the script also includes rail profile geometry in the form of cross-sections positioned along the length of the turnout. To allow for simulations of dynamic vehicle-turnout interaction in the commercial Multi Body Simulation (MBS) code Simpack [2], models are converted to Simpack's FlexTrack format. This step involves the conversion of each rail and sleeper body from the commercial FE-code Abaqus to a flexible body in Simpack format using the Craig-Bampton substructuring method [3]. The substructure contains only a selected number of retained nodal degrees of freedom in order to reduce the size of the model. The script requires the following input parameters to generate a turnout model. An example of a crossing panel generated with the script is presented in Fig. 1.

- Turnout radius and sleeper spacing

- Weight and area moment of inertias for rails and sleepers

- Stiffness and damping properties for rail pads, ballast and check rail fastenings

- Rail profile geometry parameters. The rail cross-section profiles in the switch and crossing rails are parameterized using spline shape functions

- Check rail geometry

- Finite element discretization in terms of typical element lengths and degrees of freedom 


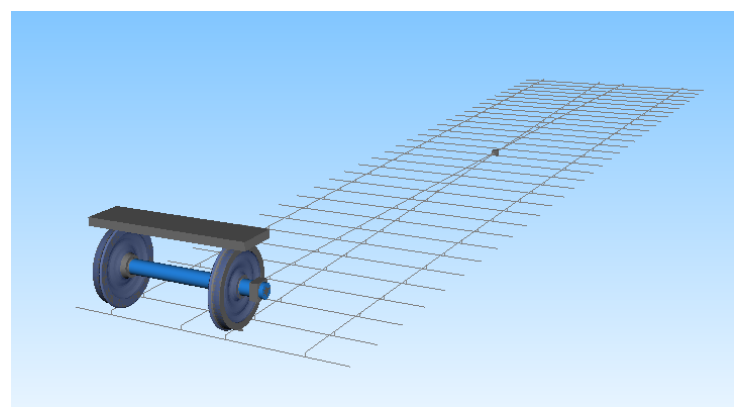

Fig. 1. Screen shot from Simpack showing a track model for a crossing panel generated via the script together with quarter vehicle model including a flexible wheelset

\section{Simulation outputs}

Wheel-rail interaction quantities such as contact forces, contact locations and contact patch sizes are available from simulations as standard outputs from Simpack. The structural response of the track for the retained degrees-of-freedom (dofs) is also readily available. The sleeper-ballast contact pressure is obtained via a postprocessing step where the force in each discrete ballast bushing is calculated and then normalized by the sleeper bottom area corresponding to that bushing element.

The bending moments for crossing rail and sleepers due to deformations in the vertical plane are also obtained in a post processing step as stress and strain recovery for flexible bodies is not available in Simpack's FlexTrack module. The nodal vertical displacements and the rotations about the axis normal to the plane of deformation are obtained as outputs from the simulations. Using the degree-of-freedom mapping between the original FE-model and the substructure and the FE properties for each component, the bending moments are computed along the length of crossing rail and sleepers. All post processing steps are performed in Matlab.

\section{Demonstration case}

The model is here demonstrated via calculation of the structural response in the crossing panel of a 60E1-R760-1:15 turnout under traffic loading. Calculations are performed as a function of crossing geometry degradation and vehicle speed for two different rail pad stiffnesses; $120 \mathrm{kN} / \mathrm{mm}$ and $1200 \mathrm{kN} / \mathrm{mm}$. The first value corresponds to an indirect fixing of the crossing rail to the sleeper with a rail pad. The second value correspond to a direct fixing with only a thin rubber mat between crossing rail and sleeper that results in a very stiff connection. The full parameter setting for the track model is presented in [4].

The structural loading is quantified via a) the maximum vertical wheel-rail contact force during the crossing transition $\mathrm{b}$ ) cross-sectional bending moments in the crossing rail and the sleeper underneath the crossing transition and c) sleeper-ballast contact pressure for the sleeper underneath the crossing transition. 
The crossing geometry degradation is represented by increased impact angles. The impact angle (or dip angle) is a measure of the change in heading direction experienced by a wheel in the vertical plane as it makes the transition from wing rail to crossing nose. The definition of the impact angle $(\beta)$ is illustrated in Fig. 2. Typically, the impact angle for wheels passing over the crossing increase as the geometry of crossing nose and wing rails change due to wear and plastic deformation. The impact angle can also vary significantly for different wheel profiles passing over the same crossing.

Further, we can expect the dynamic impact load $\Delta Q\left(\Delta Q=Q-Q_{0}\right.$, where $Q_{0}$ is the static wheel load) to be proportional to the product of the vehicle speed and the impact angle in analogy with the analytical treatment of impact loading at dipped joints [5]. For a more comprehensive treatment of impact angles at crossings, see e.g. [6].

In this study, different crossing geometries that provide different impact angles are obtained by altering the longitudinal inclinations of wing rail and crossing nose in a parameterized crossing geometry presented in [7]. A 6 mrad impact angle corresponds to a typical impact angle for a nominal crossing of the present type. The assumption is that $12 \mathrm{mrad}$ impact angle corresponds to a worn crossing geometry and $18 \mathrm{mrad}$ to a severly worn crossing geometry that has surpassed maintenance limits. An argument for the chosen impact angle ranges can be found in [8].

Traffic is represented by a quarter vehicle model with a flexible wheelset and an axle load of 20 metric tonnes.

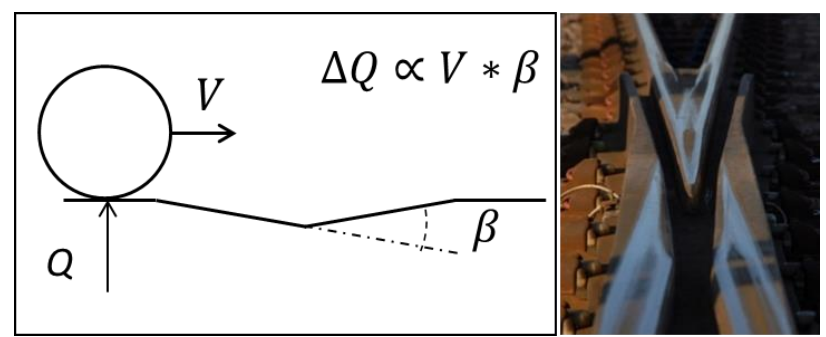

Fig. 2. (left) illustration of impact angle. (right) picture of in situ crossing rail

\section{$6 \quad$ Results}

Fig. 3 presents the maximum dynamic wheel-rail contact force during the crossing transition for different combinations of speed and impact angle for the two different pad stiffnesses. As could be expected from analytical modeling, there is a proportionality between $\Delta Q$ and the product between speed and impact angle. It can also be observed that the impact loading is slightly higher for the stiff pad configuration. 

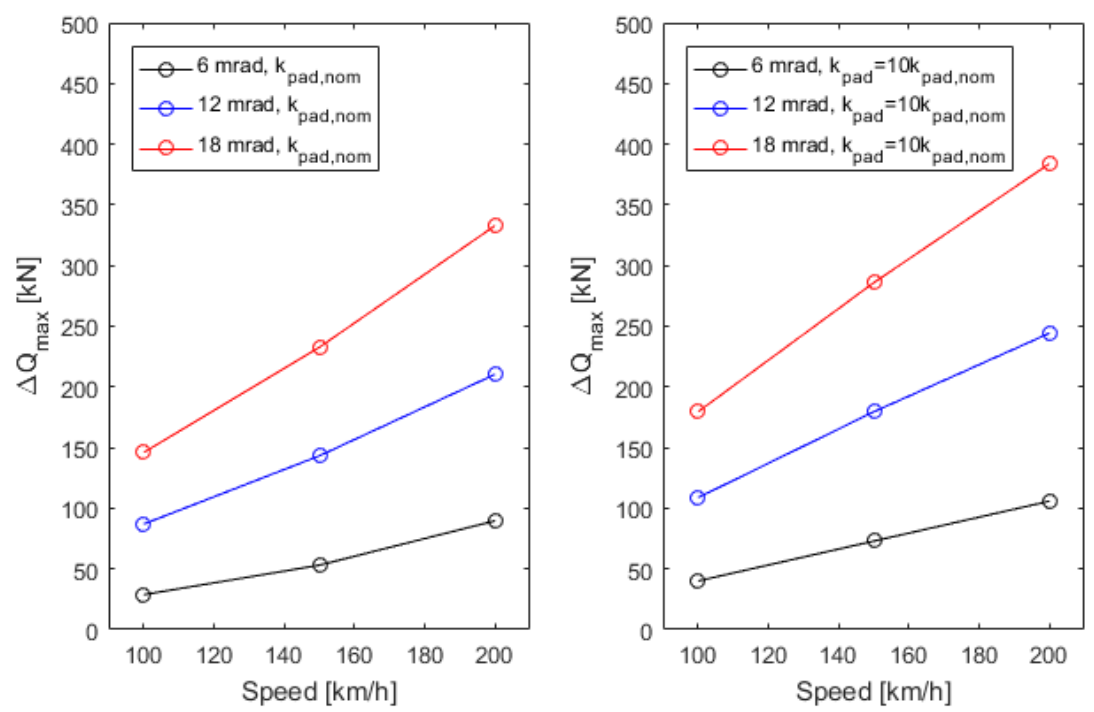

Fig. 3. Dynamic vertical impact load as a function of speed and impact angle for a soft rail pad (left, $120 \mathrm{kN} / \mathrm{mm}$ ) and a stiff rail pad (right, $1200 \mathrm{kN} / \mathrm{mm}$ )

To study the influence of traffic and track parameters on impact loading, results will first be presented for the soft rail pad. Fig. 4 presents the shape of the deformed crossing rail and the corresponding cross-sectional bending moment at the instant of maximum bending moment during the crossing transition. The results are for three different impact angles at a line speed of $200 \mathrm{~km} / \mathrm{h}$, and it can be observed that the bending moment is proportional to the impact angle. The result at $10 \mathrm{~km} / \mathrm{h}$ serves as a quasi-static reference as the dynamic contribution to the track loading at this speed is very small. The corresponding results in Fig. 5 for the sleeper under the crossing transition are qualitatively very similar to those of the crossing rail. In Fig. 5 the vertical dashed lines in the upper figure indicates the lateral positionss of the wheels of the passing wheelset.

The sleeper-ballast contact pressures are presented in Fig. 6. It can be observed that for the nominal $6 \mathrm{mrad}$ crossing geometry the model predicts a lower sleeper-ballast contact pressure under the crossing transition than for the field side of the crossing panel. It's only for the crossing geometries with a larger impact angle that the contact pressure under the crossing transition supersedes that of the field side.

Comparing the results of Fig. 5 and Fig. 6 it can also be pointed out that the maximum sleeper bending moment occurs early during the transition impact where the sleeper has mostly deformed locally under the crossing transition. The maximum sleeper-ballast contact pressure occurs later when the ballast foundation has been compressed and the sleeper has a different deformed shape.

Comparing simulation results for the two different rail pad stiffnesses it transpired that the greatest difference in loading was to be found in the sleeper bending moment. This difference in result is presented in Fig. 7. It can be observed that while the quasistatic results are more or less identical, the bending moment under the crossing transition is significantly larger with the stiffer pad. 

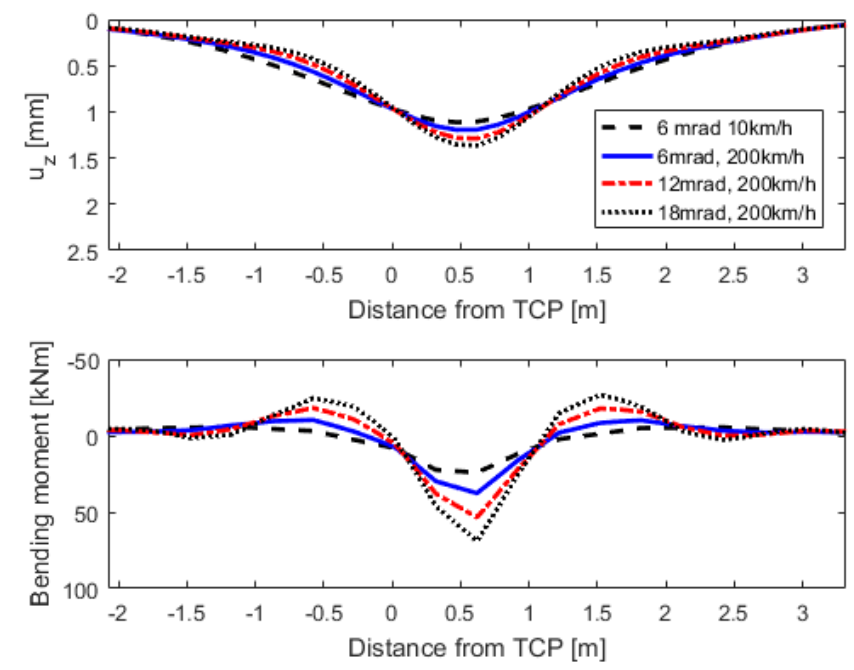

Fig. 4. Vertical displacement (top) and cross-sectional bending moment in the vertical plane (bottom) along the crossing rail. Results are for the instance with the maximum rail bending moment during the crossing transition for each simulation case. TCP=Theoretical crossing point.
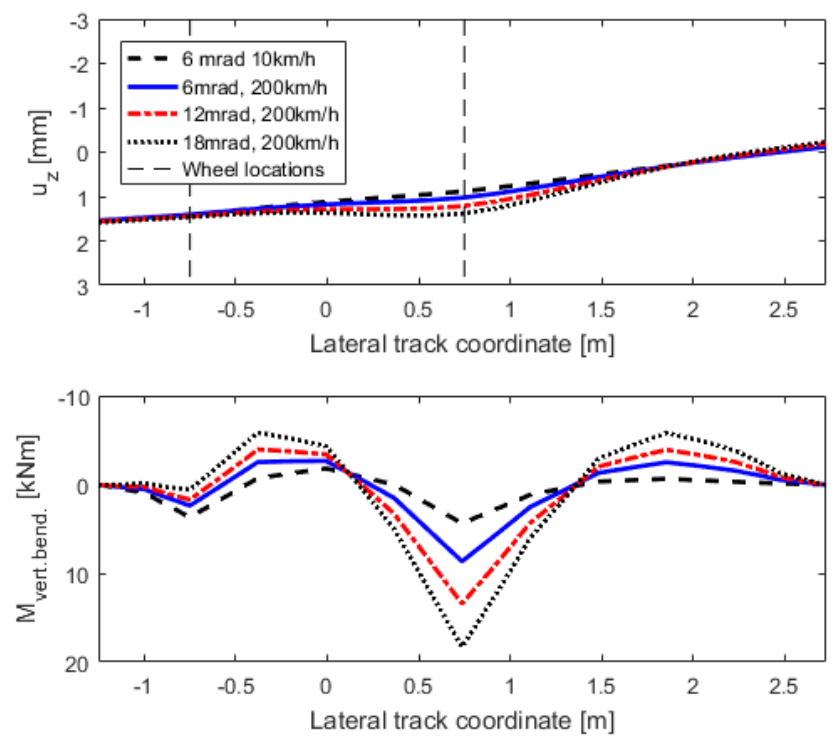

Fig. 5. Vertical displacement (top) and cross-sectional bending moment in the vertical plane (bottom) along the sleeper under the crossing transition. Results are for the instance with the maximum sleeper bending moment during the crossing transition for the different simulation cases. 

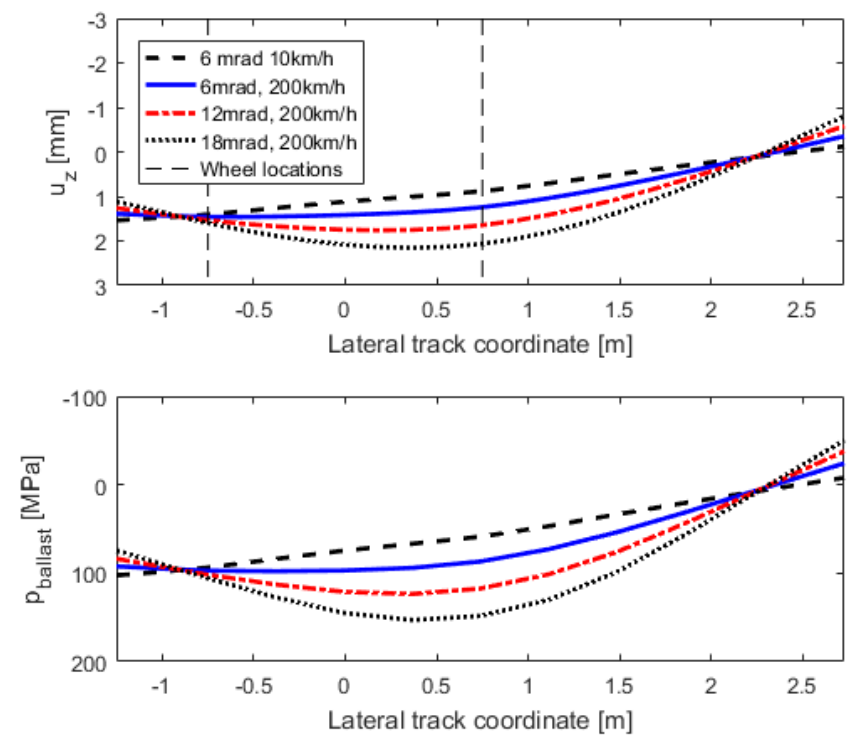

Fig. 6. Vertical displacement (top) and sleeper-ballast contact pressure (bottom) along the sleeper under the crossing transition. Results are for the instance with the maximum sleeper bending moment during the crossing transition for each simulation case.
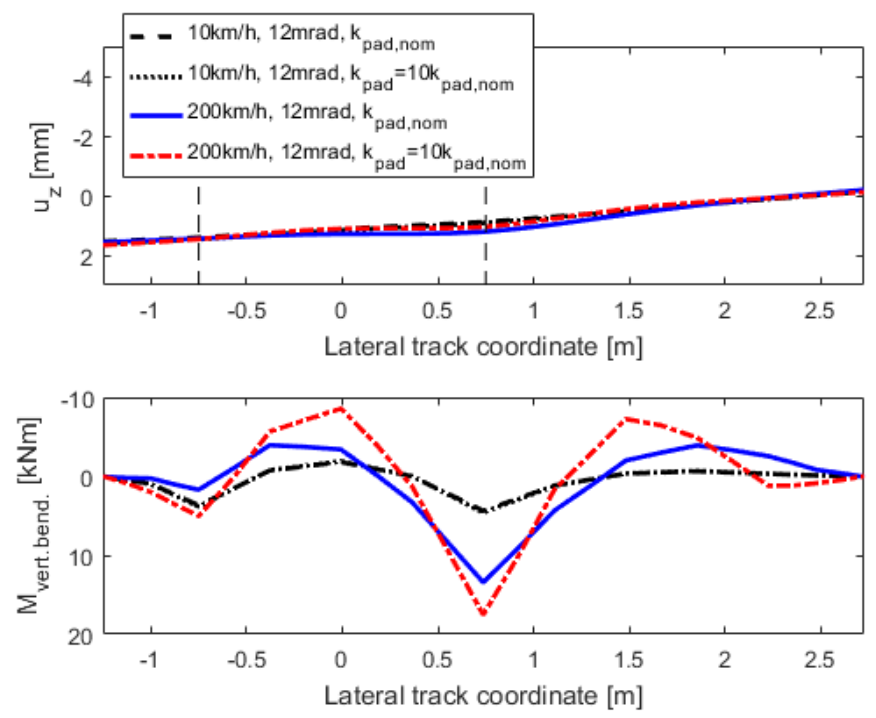

Fig. 7. Vertical displacement (top) and cross-sectional bending moment in the vertical plane (bottom) along the sleeper under the crossing transition. The results are for the instance with the maximum sleeper bending moment during the crossing transition for the different simulation cases. The lateral track coordinate is measured from the track centre line of the through route. 


\section{Conclusions}

This paper has presented a parameterized structural track model for the simulation of dynamic vehicle-turnout interaction in a multi body simulation environment. The model has been demonstrated by performing simulations and calculating the structural loading for different speeds and crossing impact angles and for different fixings between crossing rail and sleepers.

The main conclusions are that a) the peak dynamic bending moment in the sleeper under the crossing transition is significantly higher with a stiff direct fixing compared to a softer indirect fixing. b) the structural loading in terms of bending moment in the crossing rail, bending moment in the sleeper underneath the crossing transition and sleeper-ballast contact pressure increases proportionally and significantly with increased crossing impact angles.

\section{$8 \quad$ Future work}

The planned continuation of this work includes

- 3D solids representations of rails and sleepers instead of beam representations in the FE-models that form the basis for the track model. As model substructuring techniques are used to generate the structural track components in the model, the model size can still be kept at a reasonable level even if solids representations of the track components are used.

- Calculation of the expected degradation rate of the turnout structure under representative traffic loading. In particular the influence of different wheel profiles and the location of the transition between wing rail and crossing nose. The ambition is that such results could inform maintenance limits for crossing geometries.

\section{Acknowledgements}

The current study is part of the on-going activities in CHARMEC - Chalmers Railway Mechanics (www.chalmers.se/charmec). Parts of the study has been funded within the European Union's Horizon 2020 research and innovation programme in the project In2Track2 under grant agreement No 826255.

\section{References}

1. Shift2Rail homepage, https://shift2rail.org/, last accessed 2019/06/24.

2. Simpack 2019x, www.simpack.com, last accessed 2019/06/24.

3. Craig, R.R., Bampton, M.C.C.: Coupling of Substructures for Dynamic Analyses. AIAA Journal, American Institute of Aeronautics and Astronautics 6(7), 1313-1319 (1968). 
4. In2Track Deliverable D2.2, Enhanced S\&C whole system analysis, design and virtual validation, 338 pp and 2 annexes (61+11 pp), (2019)

5. Jenkins, H. H., Stephenson, J. E., Clayton, G. A., Morland, G. W., Lyon, D.: The effect of track and vehicle parameters on wheel/rail vertical dynamic forces. Railway Engineering Journal 3(1), 2-6 (1974)

6. Pålsson, B.A.: A linear wheel-crossing interaction model. Proceedings of the Institution of Mechanical Engineers Part F: Journal of Rail \& Rapid Transit 232(10), 2431-2443 (2018).

7. Pålsson, B.A.: Optimisation of railway crossing geometry considering a representative set of wheel profiles. Vehicle System Dynamics 53(2), 274-301 (2015)

8. Torstensson, P.T., Squicciarini G., Krüger M., Pålsson B.A., Nielsen, J.C.O., Thompson, D.J.: Wheel-rail impact loads and noise generated at railway crossings - Influence of vehicle speed and crossing dip angle. Journal of Sound and Vibration 456, 119-136 (2019) 